\title{
Papers
}

\section{Vitamin E supplementation and macular degeneration: randomised controlled trial}

\author{
Hugh R Taylor, Gabriella Tikellis, Luba D Robman, Catherine A McCarty, John J McNeil
}

\begin{abstract}
Objective To determine whether vitamin E supplementation influences the incidence or rate of progression of age related maculopathy (AMD). Design Prospective randomised placebo controlled clinical trial.

Setting An urban study centre in a residential area supervised by university research staff.

Participants 1193 healthy volunteers aged between 55 and 80 years; $73 \%$ completed the trial on full protocol.

Interventions Vitamin E 500 IU or placebo daily for four years.

Main outcome measures Primary outcome:

development of early age related macular degeneration in retinal photographs. Other measures included alternative definitions of age related macular degeneration, progression, changes in component features, visual acuity, and visual function

Results The incidence of early age related macular degeneration (early AMD 3) was $8.6 \%$ in those receiving vitamin $\mathrm{E}$ versus $8.1 \%$ in those on placebo (relative risk 1.05, 95\% confidence interval 0.69 to 1.61). For late disease the incidence was $0.8 \%$ versus $0.6 \%$ (1.36, 0.67 to 2.77 ). Further analysis showed no consistent differences in secondary outcomes. Conclusion Daily supplement with vitamin E supplement does not prevent the development or progression of early or later stages of age related macular degeneration.
\end{abstract}

\section{Introduction}

Age related macular degeneration (AMD) is now the leading cause of blindness and loss of vision in developed countries. ${ }^{12}$ This is due to the increased life expectancy and "greying" of the population and the successful control and treatment of other causes of blindness, such as ophthalmia neonatorum, cataract, or diabetic retinopathy. Population based studies have shown that the age specific prevalence of AMD rapidly increases in people aged over 60 years. $^{3-5}$ Two thirds of people in their $90 \mathrm{~s}$ will have $\mathrm{AMD}$, and one quarter will have the most severe form (late), which is associated with serious loss of vision. ${ }^{2}$

The cause or causes of AMD are unknown, and treatment is only partially effective and appropriate in only a few. ${ }^{6}$ There is no effective method of prevention.
A genetic basis for AMD has been suggested, ${ }^{7}$ and the genes for some similar macular disorders that occur in younger people have been described. ${ }^{8}$ Associations with several measures of cardiovascular disease and its associated risk factors are inconsistent. ${ }^{10}{ }^{11}$ Cigarette smoking is a significant risk factor for both the incidence and progression of AMD. ${ }^{4}$ Exposure to sunlight may contribute to its development, ${ }^{12}$ but this association is inconsistent. ${ }^{513}$

Many of the early changes of AMD occur at the level of the retinal pigment epithelium and failure to repair oxidative damage may be an early step in its development. Case-control and cross sectional studies have examined the association between intake or plasma concentrations of antioxidant vitamins and AMD. ${ }^{14} 15$ The findings have been inconclusive and sometimes contradictory. It is unclear whether this reflects the lack of a biological linkage or methodological difficulties in study design, dietary ascertainment, or biochemical measurements of vitamin $\mathrm{E}$.

A recent study investigated the effects of the combined antioxidant vitamins $\mathrm{A}, \mathrm{C}$, and $\mathrm{E}$ and zinc on the development of cataract and AMD. The results showed some partial protective effect of antioxidant supplements on the progression of moderately advanced AMD, but only when both eyes were affected. ${ }^{16}$ We undertook a prospective randomised controlled trial to examine whether a high dose supplement of vitamin $\mathrm{E}$ influenced the development of AMD.

\section{Methods}

\section{Study design}

One of the major arms of the vitamin E, cataract, and age related maculopathy trial (VECAT) looked at vitamin supplementation and incidence and progression of AMD. Volunteers were recruited mainly through community advertising and by post between January 1995 and April 1996. ${ }^{17}$ From the 1906 people who were screened by telephone, 1289 (69\%) were examined and 1204 (93\%) of these were enrolled and randomised. We excluded 11 participants after randomisation as they were outside the required age range at enrolment. All remaining eligible participants were those aged between 55 and 80 years in whom the lens and retina of at least one eye could be photographed. We excluded those with bilateral cataract surgery, advanced bilateral cataract, other seri-

\author{
Centre for Eye \\ Research Australia, \\ University of \\ Melbourne, Locked \\ Bag 8, East \\ Melbourne, \\ Victoria, Australia, \\ 8002 \\ Hugh R Taylor \\ professor \\ Gabriella Tikellis \\ research fellow \\ Luba D Robman \\ senior research fellow \\ Catherine A \\ McCarty \\ associate professor \\ Department of \\ Epidemiology and \\ Preventive \\ Medicine, Monash \\ University, Room \\ 306, Building ALF, \\ Alfred Hospital, \\ Caulfield, Victoria, \\ Australia, 3145 \\ John J McNeil \\ professor \\ Correspondence to: \\ H R Taylor \\ h.taylor@unimelb. \\ edu.au
}

bmj.com 2002;325:11 
ous disease, or sensitivity to vitamin $\mathrm{E}$ or who were taking steroids or anticoagulant treatment.

We obtained written informed consent. The project was approved by the standing committee on ethics in research on humans at Monash University (project 50/91).

Annual follow up examinations were planned within one month of the anniversary of enrolment. Follow up ended in January 2000. A standardised eye examination was performed that has been described in detail elsewhere. ${ }^{18}$ It included the measurement of best corrected visual acuity, visual function (VF-14), slit lamp examination, and funduscopy and photography through dilated pupils.

\section{Randomisation}

Participants randomly received either 500 IU natural vitamin $\mathrm{E}$ (335 $\mathrm{mg} \mathrm{d}-\alpha$ tocopherol) in a soybean oil suspension encapsulated in gelatin or a matched placebo capsule containing only the soybean oil. Study numbers were allocated sequentially by the study coordinator as participants were enrolled in the study. Participants were then randomly allocated to treatment group. This random allocation was performed by using a "permuted blocks" allocation scheme. The allocation list was stored at a remote site.

Bulk medications were dispensed into labelled jars by a person not involved in the study. Vitamin $\mathrm{E}$ and placebo were dispensed on different days to avoid confusion. Identical containers were used. The jars were packed in numerical order and then dispensed by study personnel.

Vitamin E and placebo capsules were of identical appearance and taste. Neither study staff nor examiners or participants were aware of the treatment allocation, although all knew that participants would be randomly assigned to receive either vitamin $\mathrm{E}$ or placebo.

The number of bottles and capsules returned by participants during the course of the study were documented as one measure of compliance. Participants were encouraged to report any adverse effects immediately and were asked specifically about adverse events and compliance during quarterly telephone calls or letters and at the annual examination. Adverse effects were investigated and periodically reported to the data monitoring and safety committee for review.

\section{Outcomes}

The primary outcome of the study was the development of early AMD (fig 1). Secondary outcomes were the progression of early AMD, the development of late AMD (fig 2), changes in visual acuity (the number of letters read on the LogMAR chart), and changes in visual function (VF-14 score).

\section{Grading of age related macular degeneration}

The clinical assessment of AMD was performed with 90 and 78 dioptre lenses and was graded according to the international classification..$^{19}$ One frame simultaneous stereophotographs of the macula were taken with a Nidek 3-DX fundus camera (Nidek, Japan) with Kodachrome 64 ASA colour film. These photographs were graded independently for AMD by trained graders according to the international classification. ${ }^{19}{ }^{20}$ Sets of circles were used to estimate drusen size and the area affected with drusen or abnormalities in retinal

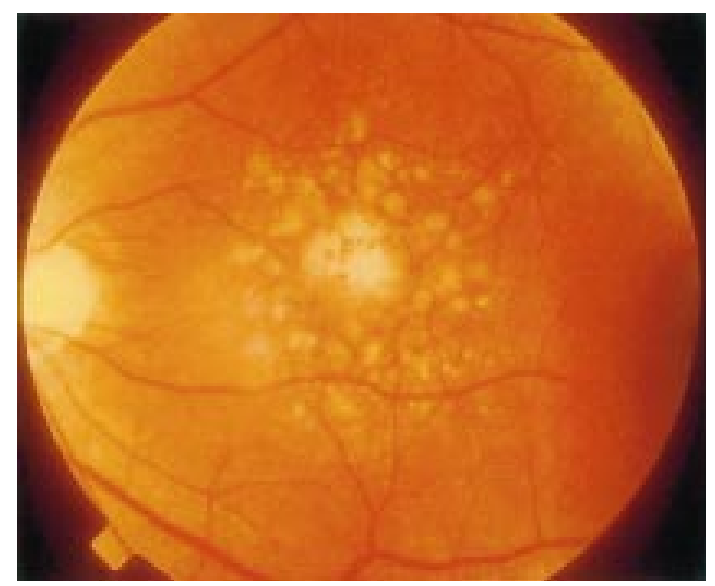

Fig 1 Early age related macular degeneration (AMD), characterised by numerous drusen of various sizes and types that extend across macular. Larger soft drusen types are of particular concern because of risk of developing into late AMD

pigmentary. At the end of the study we reassessed the initial and final photographs for any change with a "side by side" comparison in a masked and randomised fashion.

The grading of retinal photographs was performed over five years. Two people graded the baseline slides (grading was in the proportion 70:30). Slides for the remaining four years of follow up were graded by GT. To prevent systematic bias or drift we carried out retraining followed by observer trials every three months, when $10 \%$ of randomly selected slides were regraded. All cases of late AMD were also included in this masked regrading. We assessed agreement between observers by unweighted kappa statistics: $\kappa$ ranged from 0.77 (95\% confidence interval 0.30 to 1.00) for hyperpigmentation to 0.86 (0.40 to 1.00$)$ for soft distinct drusen and 1.00 for soft indistinct drusen, hypopigmentation, and late AMD. Cases in which the retinal changes were questionable or uncertain were referred to a retinal specialist for adjudication.

Because the international classification and grading system for age related maculopathy and age related

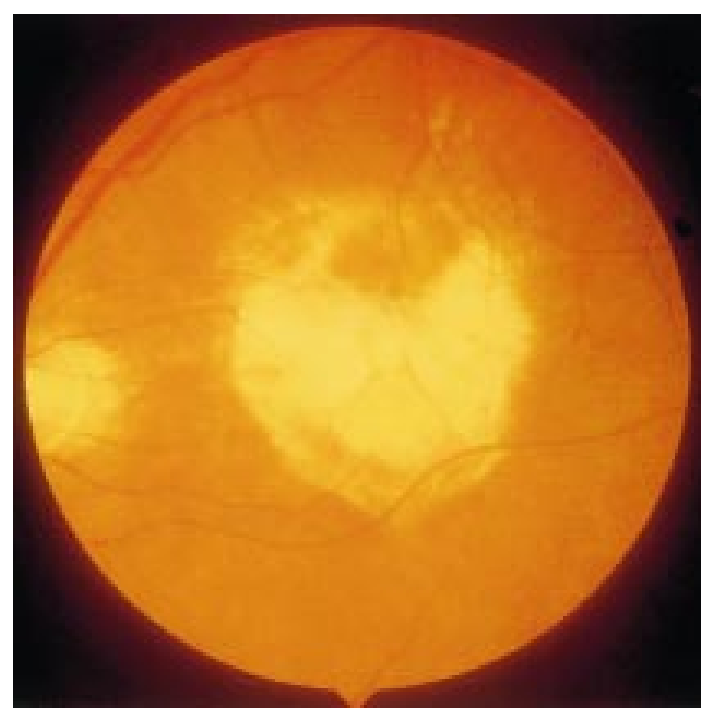

Fig 2 Late age related macular degeneration (AMD): exudative end stage. Extensive fibrovascular scar that covers macula results in severe loss of vision 
macular degeneration is somewhat ambiguous about the inclusion of soft intermediate drusen and pigmentary changes $^{4519}$ we modelled several different definitions of early AMD (table 1). Early AMD-3 was the primary outcome.

We considered that AMD progressed through six stages: (a) no drusen or only hard drusen; (b) intermediate drusen or hyperpigmentation without hypopigmentation; (c) soft drusen or pigmentary change; (d) soft drusen and pigmentary change; (e) geographic atrophy; and (f) neovascular AMD. We defined progression as movement from a lower stage to a higher stage. Participants were categorised by their worse eye.

We defined the incidence of early AMD as the appearance of early AMD in at least one eye of participants who did not have AMD in either eye at baseline.

Late AMD included neovascular AMD with serious or haemorrhagic detachment of the retinal pigment epithelium or sensory retina, characteristic haemorrhages, or subretinal fibrous scars. ${ }^{19}$ We defined atrophic late AMD as a central areolar zone of retinal pigment epithelial atrophy with visible choroidal vessels, at least $175 \mu \mathrm{m}$ in diameter, in the absence of signs of neovascular AMD in the same eye. ${ }^{19}$

\section{Sample size}

The VECAT study was initially designed to evaluate cataract, and the original estimation of sample size was based on a four year progression rate of $15 \%$ for nuclear cataract. ${ }^{21}$ One thousand people allocated in a 1:1 ratio to vitamin $\mathrm{E}$ or placebo would detect a $15 \%$ reduction (type I error of 0.01 , power 0.95 ), with allowance for a $10 \%$ annual loss due to death or withdrawals.

Given a sample size of 1000 people we calculated the power to detect changes in the incidence and progression of early AMD from published data. ${ }^{32}$ Power calculations were based on baseline data with type 1 error of 0.05 and a power of 0.80 . The final statistical power of this study to detect a $50 \%$ reduction in the incidence of early AMD (early AMD 3) was $82 \%$. The power to detect a $50 \%$ reduction in the progression of early AMD was $98 \%$. However, the power to detect a change in the incidence or progression of individual features of AMD such as soft drusen and pigment changes was lower $(\leqslant 70 \%)$.

We used the nQuery Advisor' software program, release 2.0 (Statistical Solutions, Crosse's Green, Cork, Ireland) for all power calculations.

\section{Data analysis}

Data were entered directly on to either a computer or self coding forms. Open ended responses were coded later. Double data entry was used. Data were cross checked for inconsistencies. The allocation code was not broken until all the data cleaning had been completed and the dataset "locked." Analyses were based on intention to treat. However, we performed subanalyses including only those who continued on protocol-that is, those who took the study capsules to the end of the study. We used SPSS 8.0 (SPSS, Chicago, IL) in the data analyses.

The safety committee assessed adverse events each year. The study was to be stopped if serious adverse effects occurred that were attributed to vitamin E. There was no interim analysis of the outcomes for the duration of the study.
Table 1 Definitions used to model early age related macular degeneration

\begin{tabular}{lcc} 
Feature & \multicolumn{1}{c}{ Grading of photographs } & Clinical grading \\
\hline Early AMD 1 & $\begin{array}{c}\text { Soft intermediate or soft distinct or soft } \\
\text { indistinct or pigment changes } \\
\text { (hyperpigmentation or hypopigmentation) }\end{array}$ & Not applicable \\
\hline Early AMD 2 & $\begin{array}{c}\text { Soft intermediate or soft distinct or soft } \\
\text { indistinct and pigment changes } \\
\text { (hyperpigmentation or hypopigmentation) }\end{array}$ & Not applicable \\
\hline Early AMD 3* & $\begin{array}{c}\text { Soft distinct or soft indistinct or pigment } \\
\text { changes (hyperpigmentation or } \\
\text { hypopigmentation) }\end{array}$ & $\begin{array}{c}\text { Large/soft drusen or non-geographical } \\
\text { RPE atrophy }\end{array}$ \\
\hline Early AMD 4 & $\begin{array}{c}\text { Soft distinct or soft indistinct and } \\
\text { pigment changes (hyperpigmentation or } \\
\text { hypopigmentation) }\end{array}$ & $\begin{array}{c}\text { Large/soft drusen and non-geographical } \\
\text { RPE atrophy }\end{array}$ \\
\hline
\end{tabular}

RPE=retinal pigment epithelium. ${ }^{*}$ Primary outcome.

Table 2 Comparison of baseline characteristics between two groups of participants. Figures are numbers (percentage) of participants unless otherwise stated

\begin{tabular}{|c|c|c|}
\hline \multirow[b]{2}{*}{ Characteristic } & \multicolumn{2}{|c|}{ Treatment group } \\
\hline & Vitamin E & Placebo \\
\hline Mean age (years) & 65.72 & 65.73 \\
\hline Women & $321 / 595(54)$ & $349 / 598(58)$ \\
\hline Current smokers & $14 / 589(2.3)$ & 10/597 (1.7) \\
\hline Ever smoked & $285 / 594(48)$ & 292/595 (49) \\
\hline $\begin{array}{l}\text { Best corrected visual acuity ( } \geqslant 40 \text { letters on } \\
\text { LogMAR) }\end{array}$ & 593/595 (99) & $597 / 598(99)$ \\
\hline Blue iris colour & $255 / 593(43)$ & $252 / 596(42)$ \\
\hline Cortical opacity $(\geqslant 2)$ & $97 / 595(16)$ & 69/597 (12) \\
\hline Nuclear opacity $(\geqslant 2)$ & $26 / 594(4)$ & $25 / 596(4)$ \\
\hline Any posterior subcapsular opacity & $18 / 595(3)$ & 18/597 (3) \\
\hline Mean VF-14 score & 92.5807 & 92.7431 \\
\hline Early AMD & $104 / 595(17.5)$ & $110 / 598(18)$ \\
\hline Late AMD & $3 / 595(0.5)$ & $5 / 598(0.5)$ \\
\hline \multicolumn{3}{|l|}{ Family history: } \\
\hline Cataract & 167/595 (28) & $175 / 598(29)$ \\
\hline AMD & $11 / 595(2)$ & $11 / 598(2)$ \\
\hline Glaucoma & $52 / 595(9)$ & $58 / 598(10)$ \\
\hline Blindness & $41 / 595(7)$ & $34 / 598(6)$ \\
\hline Mean visible light exposure & 21.04 & 20.14 \\
\hline Mean ultraviolet light exposure & 0.55 & 0.52 \\
\hline Previous intake of vitamin E supplementation & $156 / 553(28)$ & $133 / 542(25)$ \\
\hline Median dietary intake of vitamin E (mg/day) & 3.62 & 3.72 \\
\hline Body mass index $\geqslant 27$ & 248/595 (42) & $220 / 598(37)$ \\
\hline Hypertension & $226 / 595(38)$ & 196/598 (33) \\
\hline Hyperlipidaemia & $151 / 595(25)$ & $141 / 598(24)$ \\
\hline Ischaemic heart disease & $67 / 595(11)$ & $54 / 598(9)$ \\
\hline Diabetes & 29/595 (4.9) & 21/598 (3.5) \\
\hline
\end{tabular}

\section{Results}

\section{Characteristics of participants}

We enrolled 1193 participants (fig 3). The groups were highly comparable with no differences in baseline characteristics except for a small excess in the number with cortical lens opacities in the vitamin $\mathrm{E}$ group $\left(\chi^{2}=5.6, \mathrm{P}=0.02\right.$, table 2$)$.

The rate of compliance with the study protocol for treatment and examinations was high and similar for both groups (tables 3 and 4). In the vitamin E group eight people were excluded from final data analysis: six developed diabetic retinopathy, one had myopic degeneration, and one had missing data. Six people were excluded from the placebo group: two developed adult vitelliform macular degeneration and four had missing data.

Mean serum vitamin $\mathrm{E}$ concentrations at baseline were 39.4 (SD 20.9) $\mu \mathrm{mol} / 1$ in the vitamin E group and $35.3(6.6) \mu \mathrm{mol} / \mathrm{l}$ in the placebo group. At two and four years respectively, the concentrations were 63.8 
Table 3 Reasons for withdrawal or discontinued intervention

\begin{tabular}{|c|c|c|c|c|}
\hline & \multicolumn{2}{|c|}{ Withdrawn } & \multicolumn{2}{|c|}{ Discontinued intervention } \\
\hline & Vitamin $\mathrm{E}$ & Placebo & Vitamin $\mathrm{E}$ & Placebo \\
\hline Died & 11 & 7 & 0 & 0 \\
\hline Adverse reaction & 4 & 7 & 12 & 10 \\
\hline After cataract extraction & 1 & 1 & 2 & 2 \\
\hline Relocated & 4 & 5 & 1 & 0 \\
\hline Health related & 24 & 21 & 13 & 7 \\
\hline Personal & 23 & 24 & 8 & 12 \\
\hline Wanted to take own vitamin $\mathrm{E}$ & 4 & 1 & 12 & 9 \\
\hline Contraindication to vitamin $\mathrm{E}$ & 4 & 3 & 12 & 11 \\
\hline Changed studies & 0 & 0 & 0 & 1 \\
\hline Unknown & 3 & 3 & 14 & 18 \\
\hline Total & 78 & 72 & 74 & 70 \\
\hline
\end{tabular}

Table 4 Annual status of study participants

\begin{tabular}{lccccccc} 
& \multicolumn{3}{c}{ Vitamin $\mathrm{E}$} & & \multicolumn{3}{c}{ Placebo } \\
\cline { 2 - 4 } \cline { 7 - 8 } Time point & On protocol & Off protocol* $^{*}$ & Withdrawn & & On protocol & Off protocol $^{*}$ & Withdrawn \\
\hline Baseline & 595 & 0 & 0 & & 598 & 0 & 0 \\
\hline 1 year & 533 & 34 & 28 & & 525 & 33 & 40 \\
\hline 2 years & 497 & 44 & 54 & & 496 & 48 & 54 \\
\hline 3 years & 468 & 58 & 69 & & 483 & 51 & 64 \\
\hline 4 years & 443 & 74 & 78 & & 456 & 70 & 72 \\
\hline
\end{tabular}

*Off protocol indicates ceased study medication but underwent annual examination.

(21.6) $\mu \mathrm{mol} / \mathrm{l}$ and $58.3(25.6) \mu \mathrm{mol} / \mathrm{l}$ in the vitamin $\mathrm{E}$ group and 32.0 (SD 8.8) $\mu \mathrm{mol} / \mathrm{l}$ and 31.4 (SD 8.9) $\mu \mathrm{mol} / \mathrm{l}$ for the placebo group (at two years Student's $t=6.82, \mathrm{P}>0.001$; at four years $t=4.98, \mathrm{P}>0.001)$.

We assessed compliance by counting left over capsules, and $78 \%$ of participants had a compliance rate of $80 \%$ or higher based on intention to treat analyses. There was no difference in compliance between the two groups $\left(\chi^{2}=3.61, \mathrm{df}=1, \mathrm{P}=0.46\right)$. We measured plasma concentrations in a subgroup of participants to assess compliance with treatment allocation.

\section{Adverse events}

We classified adverse events according to the body system affected. No serious adverse events were reported, though 678 people reported at least one adverse event.

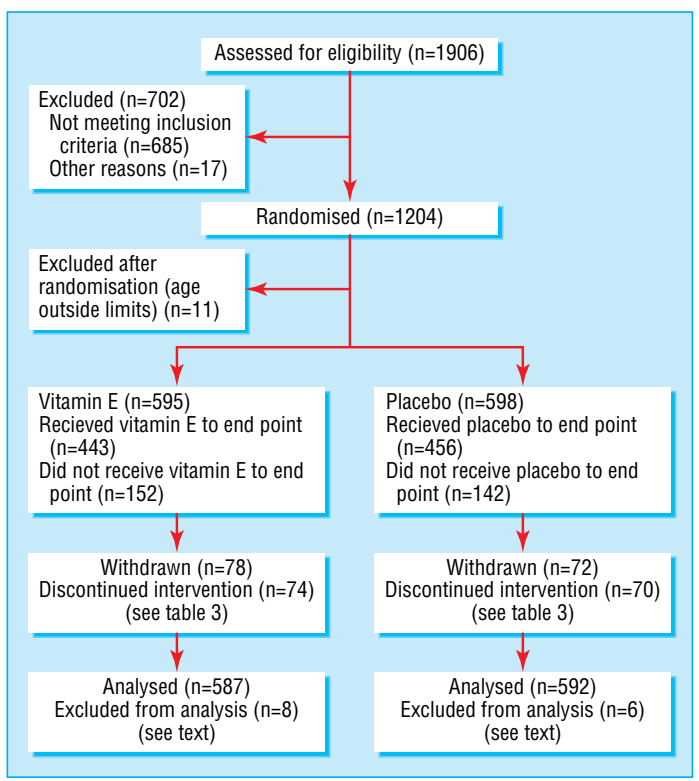

Fig 3 Randomisation of participants for vitamin E, cataract, and age related maculopathy (VECAT) study
There was no significant difference between overall number and type of adverse event between the two groups $\left(\chi^{2}=1.82, \mathrm{df}=7, \mathrm{P}=0.97\right)$. A total of 174 adverse events were potentially related to the use of study capsules, $91(15 \%)$ in the vitamin E group and $83(14 \%)$ in the placebo grpup $\left(\chi^{2}=0.48, \mathrm{df}=1, \mathrm{P}=0.49\right)$. Ophthalmic adverse events were reported by $105(18 \%)$ in the vitamin E group and $90(15 \%)$ in the placebo group $\left(\chi^{2}=1.44, \mathrm{df}=1, \mathrm{P}=0.23\right)$.

\section{Early AMD}

Incidence-There was no difference in the four year incidence of early AMD in the two treatment groups over the four years (table 5). This was true for each definition tested and for both grading of photographs and clinical grading. Similarly, there was no difference between the incidence of the separate features of early AMD and treatment, except for hypopigmentation. Hypopigmentation was significantly less common in those on vitamin $\mathrm{E}$, although the clinical significance of this is unclear. In addition, there were no differences between the groups in the prevalence of early AMD, its component features, or late AMD at baseline or at four years by either grading of photographs or clinical grading (tables 6 and 7).

Progression-According to grading of photographs 95 of $491(19 \%)$ in the vitamin $\mathrm{E}$ group showed progression compared with 90 of $506(18 \%)$ in the placebo group (relative risk=1.09, 0.84 to 1.42 ). By clinical grading, we observed progression in 40 of $508(7.9 \%)$ in the vitamin E group and 31 of $514(6.0 \%)$ in the those placebo group $(1.31,0.83$ to 2.07$)$. We saw no difference in the rate of progression of drusen types by treatment group (hard drusen being replaced by soft drusen, intermediate soft drusen being replaced by soft distinct or indistinct drusen, or the increase in area of either soft distinct or soft indistinct drusen; data not shown).

A masked "side by side" comparison of photographs from baseline and at four years showed no significant difference between the two groups (table 8). There was slightly more progression in the vitamin $\mathrm{E}$ group, which was only marginally significant $(1.26,1.01$ to 1.57$)$.

\section{Additional analysis}

Analysis of best corrected visual acuity and visual function data showed no differences between the groups (data not shown). Similar numbers of people lost more than nine letters (two lines) of visual acuity (59 in vitamin E group, 57 in placebo group).

Further analyses included all cases of AMD, geographic atrophy alone, and neovascular AMD alone. Subgroup analyses included current smokers, those with a family history of AMD, and those with a high ocular exposure to visible light or to ultraviolet-B radiation. In none of these analyses was there a difference between the two treatment groups. Similarly, no difference was found when we repeated the analyses and controlled for the baseline presence of cortical lens opacities.

Finally, a multiple logistic regression analysis that included potential confounders of incidence or progression showed no association between the study intervention and the incidence or progression of early AMD. 
Table 5 Four year incidence* of features related to early AMD, AMD, and late AMD. Figures are numbers (percentage) of participants with feature and risk ratios ( $95 \%$ confidence interval)

\begin{tabular}{|c|c|c|c|c|c|c|}
\hline & \multicolumn{3}{|c|}{ Grading of photographs } & \multicolumn{3}{|c|}{ Clinical grading } \\
\hline & Vitamin E & Placebo & Risk ratio $(95 \% \mathrm{CI})$ & Vitamin E & Placebo & Risk ratio $(95 \% \mathrm{Cl})$ \\
\hline Soft intermediate drusen & 78/403 (19) & 73/397 (18) & 1.05 (0.80 to 1.39$)$ & NA & NA & NA \\
\hline Soft distinct drusen & $29 / 482(6)$ & $28 / 487(6)$ & 1.05 (0.60 to 1.82$)$ & NA & NA & NA \\
\hline Soft indistinct drusen & $7 / 451(2)$ & $7 / 466(2)$ & 1.03 (0.77 to 1.38) & NA & NA & NA \\
\hline Hypopigmentation & $6 / 470(1)$ & $16 / 481(3)$ & 0.38 (1.16 to 0.93$)$ & NA & NA & NA \\
\hline Hyperpigmentation & $21 / 459(5)$ & $32 / 470(7)$ & 0.68 (0.41 to 1.14$)$ & NA & NA & NA \\
\hline Early AMD 1 & $79 / 339(23)$ & $70 / 330(21)$ & $1.10(0.82$ to 1.47$)$ & NA & NA & NA \\
\hline Early AMD 2 & $10 / 480(2)$ & $14 / 489(3)$ & 0.73 (0.33 to 1.62$)$ & NA & NA & NA \\
\hline Early AMD $3 \dagger$ & $35 / 409(9)$ & $34 / 418(8)$ & 1.05 (0.69 to 1.61) & $28 / 387(7)$ & $25 / 386(7)$ & $1.12(0.66$ to 1.90$)$ \\
\hline Early AMD 4 & $6 / 488(1)$ & 9/498 (2) & 0.68 (0.25 to 1.88$)$ & $9 / 495(1)$ & $5 / 495(1)$ & 0.56 (0.20 to 1.64$)$ \\
\hline Late AMD & $4 / 494(1)$ & $3 / 504(1)$ & 1.36 (0.67 to 2.77$)$ & $3 / 507(1)$ & $3 / 510(1)$ & $1.00(\mathrm{NA})$ \\
\hline
\end{tabular}

$\mathrm{NA}=$ Not applicable to clinical grading.

*Incidence=absence of particular lesion at baseline and presence of this lesion in at least one eye at four years.

tPrimary outcome.

Table 6 Prevalence of early and late AMD assessed by photograph grading at baseline and at four years. Figures are numbers (percentage) of participants

\begin{tabular}{|c|c|c|c|c|c|c|c|c|}
\hline & \multicolumn{4}{|c|}{ Baseline } & \multicolumn{4}{|c|}{ Four years } \\
\hline & Vitamin E & Placebo & $\chi^{2}(\mathrm{df}=1)$ & $P$ value & Vitamin E & Placebo & $\chi^{2}(\mathrm{df}=1)$ & $P$ value \\
\hline Early AMD 1 & $192 / 587(33)$ & 205/593 (35) & 0.46 & 0.50 & 167/504 (33) & $155 / 512(30)$ & 0.96 & 0.33 \\
\hline Early AMD 2 & $20 / 587$ (3) & $24 / 593(4)$ & 0.38 & 0.56 & 14/504 (3) & 22/512 (4) & 1.72 & 0.19 \\
\hline Early AMD 3 & $104 / 587$ (18) & $111 / 593(19)$ & 0.20 & 0.66 & $87 / 504$ (17) & $88 / 512(17)$ & 0.01 & 0.98 \\
\hline Early AMD 4 & 11/587 (2) & 11/593 (2) & 0.06 & 0.98 & 7/504 (1) & 10/512 (2) & 0.49 & 0.48 \\
\hline Late AMD & $3 / 587(0)$ & 4/593 (1) & NA & $1.00^{*}$ & $5 / 504(1)$ & 4/512 (1) & NA & $0.75^{\star}$ \\
\hline
\end{tabular}

NA=not applicable.

${ }^{*}$ Fisher's exact test.

Table 7 Prevalence of early and late AMD as assessed by clinical grading at baseline and at four years. Figures are numbers (percentage) of participants

\begin{tabular}{|c|c|c|c|c|c|c|c|c|}
\hline & \multicolumn{4}{|c|}{ Baseline } & \multicolumn{4}{|c|}{ Four years } \\
\hline & Vitamin E & Placebo & $\chi^{2}(\mathrm{df}=1)$ & $P$ value & Vitamin E & Placebo & $\chi^{2}(\mathrm{df}=1)$ & $P$ value \\
\hline Early AMD 3 & $151 / 595(25)$ & $149 / 598$ (25) & 0.03 & 0.85 & $104 / 508(21)$ & 95/514 (19) & 0.65 & 0.42 \\
\hline Early AMD 4 & $19 / 595$ (3) & $18 / 598(3)$ & 0.03 & 0.86 & $14 / 508(3)$ & $14 / 514(3)$ & 0.01 & 0.98 \\
\hline Late AMD & 3/508 (1) & 4/593 (1) & NA & $1.00^{*}$ & 4/508 (1) & 4/514 (1) & NA & $1.00^{*}$ \\
\hline
\end{tabular}

$\mathrm{NA}=$ not applicable.

${ }^{\star}$ Fisher's exact test.

\section{Discussion}

In this four year study of the effect of vitamin E supplementation of the development and progression of early age related macular degeneration (early AMD-3) we found no protective or deleterious effect of the daily dietary supplementation of $500 \mathrm{IU}$ vitamin $\mathrm{E}$ on incidence or progression. Further analysis of the incidence of individual features of early AMD also showed no protective effect of supplementation except for a decrease in retinal hypopigmentation. However, the clinical significance of this finding is unclear and it may be due to chance alone. The secondary analyses of visual acuity and visual function also failed to show an intervention effect.

To our knowledge this is the first prospective randomised controlled trial to evaluate vitamin E supplementation and age related macular degeneration.

Table 8 Progression as determined by "side by side" comparison of baseline and four year photographs. Figures are numbers (percentage) of participants

\begin{tabular}{lcc} 
& Vitamin E & Placebo \\
\hline Better & $20 / 493(4)$ & $28 / 505(6)$ \\
\hline Same & $337 / 493(68)$ & $366 / 505(73)$ \\
\hline Worse & $136 / 493(28)$ & $111 / 505(22)$ \\
\hline
\end{tabular}

The strengths of this study include the sample size, the high rates of compliance and follow up, the prospective and randomised design, and photographic documentation. Weaknesses in the study were the relatively short follow up (four years) and the relatively low proportion of cigarette smokers.

We saw no deleterious effects associated with vitamin $\mathrm{E}$ supplementation, and the adverse effects that were reported occurred with a similar frequency in each group. This is consistent with data from another recent report about vitamin E supplementation. ${ }^{23}$

The physicians health study showed that physicians who used either vitamin $\mathrm{E}$ or multivitamins had a $13 \%$ and $10 \%$ reduction in the risk of AMD respectively, although this finding was not significant. ${ }^{24}$ Since we completed our study results from the age related eye disease study (AREDS) have become available. ${ }^{16}$ That study examined the effect of a combination of antioxidants (vitamin C 500 mg/day; vitamin E 400 IU/day; $\beta$ carotene $15 \mathrm{mg} /$ day) with and without zinc ( $80 \mathrm{mg}$ as zinc oxide and copper and $2 \mathrm{mg}$ as cupric oxide). They found a reduction in the progression of photographic signs of AMD but only in those with moderately advanced disease in both eyes. They found no effect on earlier or later stages of AMD or in those with 


\section{What is already known on this topic}

Age related macular degeneration is the leading cause of loss of vision and blindness in elderly people; for people aged $\geqslant 90$ years, two out of every three will be affected and one in four will become blind

Currently, there are no methods of prevention or treatment in most cases, though a third of cases are due to cigarette smoking

Antioxidant vitamins have been suggested as a possible prevention

\section{What this study adds}

Daily supplementation with $500 \mathrm{mg}$ vitamin $\mathrm{E}$ for four years did not alter the incidence or progression of AMD

unilateral disease. They did not examine the effect of vitamin $\mathrm{E}$ supplementation on its own.

\section{Implications}

The lack of a protective effect of vitamin E supplementation is somewhat disappointing. Possibly our follow up period was too short and vitamin $\mathrm{E}$ may need to be taken for a long time, if not for the whole of life, or in combination with other antioxidants. There may be a long time lag between the time of damage and the appearance of clinical signs. In addition, antioxidants may be effective only in certain subgroups of people who are at particular risk or who have a high exposure to retinal damage or oxidation, such as those with a genetic susceptibility, cigarette smokers, or those with a high ocular light exposure. ${ }^{25}$ Alternatively, our findings may mean that vitamin $\mathrm{E}$ does not have an important role in protecting against macular degeneration. This last conclusion would be consistent with the variable and often contradictory results obtained from previous cross sectional studies.

Contributors: HRT, GT, LDR, JJM, and CAM wrote the paper. JJM, HRT, Martha Sinclair, and the late Chris Silagy designed the study. JJM, HRT, CAM, LDR, Sinead K Garrett, MS, Flavia Cicuttini, Kath Ogden, and Adrian Thomas were members of the study's steering committee. The Data Safety and Monitoring Committee was chaired by the late Professor Chris Silagy. IJM, HRT, CAM were overall supervisors for the VECAT study. CAM was the trial epidemiologist. SKG was study coordinator. Hugo Stephenson designed and managed the software used for data collection. GT, LDR, and SKG conducted the study. Alex Harper was the external retinal consultant. LDR was the study ophthalmologist, was responsible for all fundus photography and clinical eye examinations, and was internal adjudicator for fundus grading. GT was responsible for all macular grading, development of grading protocol, participant interviews and medical examinations, and assessment of compliance to study protocol. Lynne Rodereda and Trudy Mai contributed to the data collection. LDR, GT, Kath Ogden, and Nicole Doherty contributed to the finalisation of the dataset. GT, LDR, and CAM (statistical supervisor) were responsible for data analysis. HRT is guarantor.

Funding: The VECAT study was funded in part by grants from the National Health and Medical Research Council, the
Jack Brockhoff Foundation, the Eirene Lucas Foundation, the Stoicesco Foundation, the Carleton Family Charitable Trust, Je Hope Knell Trust Fund, Smith and Nephew, Australia, and Henkel Australia.

Competing interests: None declared.

1 Evans J, Wormald R. Is the incidence of registrable age-related macular degeneration increasing? Br J Ophthalmol 1996;80:9-14.

2 VanNewkirk M, Weih LM, McCarty CA, Taylor HR. Cause-specific prevalence of bilateral visual impairment in Victoria, Australia: the visual impairment project. Ophthalmol 2001;108:960-7.

3 Klein R, Klein BEK, Jensen SC, Meuer SM. The five-year incidence and progression of age-related maculopathy: the Beaver Dam eye study. Ophthalmology 1997;104:7-2

4 Smith W, Assink J, Klein R, Mitchell P, Klaver CC, Klein BE, et al. Risk factors for age-related macular degeneration: pooled findings from three continents. Ophthalmology 2001;108:697-704.

5 McCarty CA, Mukesh BN, Fu CL, Mitchell P, Wang SS, Taylor HR. Risk factors for age-related maculopathy: the visual impairment project. Arch Ophthalmol 2001;119:1455-62

6 Bressler NM. Age related macular degeneration. New hope for a common problem comes from photodynamic therapy. BMJ 2000;321:1425-7.

7 Heiba IM, Elston RC, Klein BE, Klein R. Sibling correlations and segregation analysis of age-related maculopathy: the Beaver Dam study. Genet Epidemiol 1994;11:51-67.

8 Allikmets R, Shroyer NF, Singh N, Seddon JM, Lewis RA, Bernstein PS, et al. Mutation of the Stargardt disease gene (ABCR) in age-related macular degeneration. Science 1997;227:1805-7.

Stone EM, Lotery AJ, Munier FL, Heon E, Piguet B, Guymer RH, et al. A single EFEMP1 mutation associated with both Malattia Leventinese and Doyne honeycomb retinal dystrophy. Nat Genet 1999;22:199-202.

10 Hyman LG, Lilienfeld AM, Ferris FL 3rd, Fine SL. Senile macular degeneration: a case-control study. Am J Epidemiol 1983;118:213-27.

11 Vingerling JR, Dielemans I, Bots ML. Age-related macular degeneration is associated with atherosclerosis: the Rotterdam study. Am J Epidemiol $1995 ; 142: 404-9$

12 Taylor HR, West S, Munoz B, Rosenthal FS, Bressler SB, Bressler NM. The long-term effects of visible light on the eye. Arch Ophthalmol 1992;110:99 104

13 Mitchell P, Smith W, Wang IJ. Iris color, skin sun sensitivity, and age-related maculopathy. The Blue Mountains eye study. Ophthalmology 1998; 105:1359-63.

14 Delcourt C, Cristol JP, Tessier F, Leger CL, Descomps B, Papoz L. Age-related macular degeneration and antioxidant status in the POLA study. Arch Ophthalmol 1999;117:1384-90.

15 West S, Vitale S, Hallfrisch J, Munoz B, Muller D, Bressler S, Bressler NM Are antioxidants or supplements protective for age-related macular degeneration? Arch Ophthalmol 1994;112:222-7.

16 Age-Related Eye Disease Study Research Group. A randomized, placebocontrolled, clinical trial of high-dose supplementation with vitamins $\mathrm{C}$ and $\mathrm{E}$ and beta carotene for age-related cataract and vision loss. Arch Ophthalmol 2001;119:1439-52.

17 Garrett SKM, Thomas AP, Cicuttini F, Silagy C, Taylor HR, McNeil JJ Community-based recruitment strategies for a longitudinal interventional study: the VECAT experience. J Clin Epidemiol 2000;53:541-8.

18 Robman LD, Tikellis G, Garrett SKM, Harper CA, McNeil JJ, Taylor HR, et al. Baseline ophthalmic findings in the vitamin $\mathrm{E}$, cataract and age-related maculopathy (VECAT) study. Aust $N$ Z J Ophthalmol 1999;27:410-6.

19 Bird AC, Bressler NM, Bressler SB, Chisholm IH, Coscas G, Davis MD, et al. The International ARM Epidemiological Study Group. An international classification and grading system for age-related maculopathy and age-related macular degeneration. Surv Ophthalmol 1995;39:36774

20 Tikellis G, Robman LD, Harper A, McNeil JJ, Taylor HR, McCarty C. Methods for detecting age-related maculopathy: a comparison between photographic and clinical assessment. Clin Experiment Ophthalmol 2000;28:367-72

21 West S, Munoz B, Schein OD, Vitale S, Maguire M, Taylor HR, et al. Cigarette smoking and risk for progression of nuclear opacities. Arch Ophthalmol 1995;113:1377-80.

22 Bressler NM, Muñoz B, Maguire MG, Vitale SE, Schein OD, Taylor HR, et al. Five-year incidence and disappearance of drusen and retinal pigment epithelial abnormalities: Waterman study. Arch Ophthalmol 1995;113:301-8.

23 Yusuf S, Dagenais G, Pogue J, Bosch J, Sleight P. Heart Outcomes Prevention Evaluation Study Investigators. Vitamin E supplementation and cardiovascular events in high-risk patients. N Engl J Med 2000;342:15460.

24 Christen WG, Ajani UA, Glynn RJ, Manson JE, Schaumberg DA, Chew EC, et al. Prospective cohort study of antioxidant vitamin supplement use and the risk of age-related maculopathy. Am J Epidemiol 1999;149:476-84.

25 Meagher EA, Barry OP, Lawson JA, Rokach J, Fitzgerald GA. Effects of vitamin $\mathrm{E}$ on lipid peroxidation in healthy persons. JAMA 2001;285:1178-82

(Accepted 9 January 2002) 